ANNALES

POLONICI MATHEMATICI

$84.3(2004)$

\title{
Note on the Jacobian condition and the non-proper value set
}

\author{
by NGuYen Van Chau (Hanoi)
}

\begin{abstract}
We show that the non-proper value set of a polynomial map $(P, Q)$ : $\mathbb{C}^{2} \rightarrow \mathbb{C}^{2}$ satisfying the Jacobian condition $\operatorname{det} D(P, Q) \equiv$ const $\neq 0$, if non-empty, must be a plane curve with one point at infinity.
\end{abstract}

1. Let $f=(P, Q): \mathbb{C}_{(x, y)}^{2} \rightarrow \mathbb{C}_{(u, v)}^{2}$ be a dominant polynomial map, $P, Q \in \mathbb{C}[x, y]$, and define $J(P, Q):=P_{x} Q_{y}-P_{y} Q_{x}$. Recall that the socalled non-proper value set $A_{f}$ of $f$ consists of all points $a \in \mathbb{C}^{2}$ such that the inverse $f^{-1}(K)$ is not compact for any compact neighborhood $K \subset \mathbb{C}^{2}$ of $a$. This set $A_{f}$, if non-empty, must be a plane curve such that each of its irreducible components can be parameterized by a non-constant polynomial map from $\mathbb{C}$ into $\mathbb{C}^{2}$ (see $[\mathrm{J}]$ ). The mysterious Jacobian conjecture (see $[\mathrm{BCW}]$ and $[\mathrm{E}])$, posed first by Keller in 1939 and still open, asserts that a polynomial map $f=(P, Q)$ of $\mathbb{C}^{2}$ with $J(P, Q) \equiv$ const $\neq 0$ must have a polynomial inverse. This conjecture can be reduced to proving that the non-proper value set $A_{f}$ is empty. Anyway one may think that in a counterexample to the Jacobian conjecture, if one exists, the non-proper value set must have a very special form. In $[\mathrm{C}]$ it was observed that in such a counterexample the irreducible components of $A_{f}$ can be parameterized by polynomial maps $\xi \mapsto(p(\xi), q(\xi))$ with $\operatorname{deg} p / \operatorname{deg} q=\operatorname{deg} P / \operatorname{deg} Q$. In this paper we notice that the non-proper value set of a nonsingular polynomial map from $\mathbb{C}^{2}$ into itself, if non-empty, must be a curve with one point at infinity.

Theorem 1. Suppose $f=(P, Q)$ is a polynomial map of $\mathbb{C}^{2}$ with $J(P, Q)$ $\equiv$ const $\neq 0, \operatorname{deg} P=\operatorname{deg}_{y} P=K d$ and $\operatorname{deg} Q=\operatorname{deg}_{y} Q=K e, \operatorname{gcd}(d, e)$ $=1$,

2000 Mathematics Subject Classification: Primary 14R15.

Key words and phrases: non-proper value set, Jacobian condition.

Supported in part by the National Basic Program on Natural Science, Vietnam, and the associate program of ICTP, Trieste, Italy. 


$$
\begin{array}{ll}
P(x, y)=A y^{K d}+\cdots+a_{1}(x) y+a_{0}(x), & A \neq 0, \\
Q(x, y)=B y^{K e}+\cdots+b_{1}(x) y+b_{0}(x), & B \neq 0 .
\end{array}
$$

If the non-proper value set $A_{f}$ is not empty, then every irreducible component of $A_{f}$ can be parameterized by polynomial maps of the form

$$
\begin{aligned}
& \xi \mapsto\left(A \xi^{m d}+\text { lower order terms in } \xi\right. \\
& \left.\qquad B \xi^{m e}+\text { lower order terms in } \xi\right), \quad m \in \mathbb{N} .
\end{aligned}
$$

By definition $A_{f}$ is the set of all values $a \in \mathbb{C}^{2}$ such that the number of solutions counted with multiplicities of the equation $f(x, y)=a$ is different from those for generic values in $\mathbb{C}^{2}$. Then, considering the components $P(x, y)$ and $Q(x, y)$ as elements of $\mathbb{C}[x][y]$, we can define the resultant

$$
\operatorname{Res}_{y}(P-u, Q-v)=R_{0}(u, v) x^{N}+\cdots+R_{N}(u, v),
$$

where $R_{i} \in \mathbb{C}[u, v], R_{0} \neq 0$. From the basic properties of the resultant function we know that $N$ is the geometric degree of $f$ and $A_{f}=\{(u, v) \in$ $\left.\mathbb{C}^{2}: R_{0}(u, v)=0\right\}$. Note that a curve given by a polynomial parameter of the form (1) can be defined by a polynomial of the form $\left(A^{e} u^{e}-B^{d} v^{d}\right)^{m}+$ $\sum_{0 \leq i d+j e<m d e} c_{i j} u^{i} v^{j}$ and its branch at infinity has a Newton-Puiseux series of the form $u=c v^{d / e}+$ lower order terms in $v$, where $c$ is a $d$ th root of $B^{d} / A^{e}$. Thus, Theorem 1 leads to

Corollary 1. Let $f$ be as in Theorem 1. Then

$$
R_{0}(u, v)=C\left(A^{e} u^{e}-B^{d} v^{d}\right)^{M}+\sum_{0 \leq i d+j e<M d e} c_{i j} u^{i} v^{j}
$$

with $0 \neq C \in \mathbb{C}$ and $M \geq 0$.

Corollary 2. Let $f$ be as in Theorem 1 . If $A_{f} \neq \emptyset$, then $A_{f}$ is a curve with one point at infinity and the irreducible branches at infinity of $A_{f}$ have Newton-Puiseux series of the form

$$
u=c v^{d / e}+\text { lower order terms in } v
$$

with coefficients $c$ being dth roots of $B^{d} / A^{e}$.

As seen later, the representation in (1) of $P$ and $Q$ is only used to visualize the coefficient $B^{d} / A^{e}$. In fact, when $A_{f} \neq \emptyset$ the numbers $d, e$, $B^{d} / A^{e}$ and the polynomial $R_{0}(u, v)$ are invariant under right actions of automorphisms of $\mathbb{C}^{2}$, since the set $A_{f}$ does not depend on the coordinate $(x, y)$. Furthermore, the coefficient $B^{d} / A^{e}$ is uniquely determined from the relation

$$
P_{+}^{e}(x, y)=\left(B^{d} / A^{e}\right) Q_{+}^{d}(x, y),
$$

which is a consequence of the Jacobian condition when $\operatorname{deg} P>1$ and $\operatorname{deg} Q>1$. Here, $P_{+}$and $Q_{+}$are the leading homogeneous components of $P$ and $Q$, respectively. 
Theorem 1 will be proved in Sections $2-5$ in an elementary way by using Newton-Puiseux expansions and the Newton theorem. It would be interesting to determine the form of $R_{0}(u, v)$ by examining directly the resultant function $\operatorname{Re}_{y}(P-u, Q-v)$.

2. Dicritical series of $f$. From now on, $f=(P, Q): \mathbb{C}_{(x, y)}^{2} \rightarrow \mathbb{C}_{(u, v)}^{2}$ is a given polynomial map with $J(P, Q) \equiv$ const $\neq 0, \operatorname{deg} P=K d>0$ and $\operatorname{deg} Q=K e>0, \operatorname{gcd}(d, e)=1$. The Jacobian condition will be used really in Lemma 3 and the proof of Theorem 1. Since $A_{f}$ does not depend on the coordinate $(x, y)$, to examine it we can assume that $\operatorname{deg}_{y} P=\operatorname{deg} P$, $\operatorname{deg}_{y} Q=\operatorname{deg} Q$ and

$$
\begin{array}{ll}
P(x, y)=A y^{K d}+\cdots+a_{1}(x) y+a_{0}(x), & A \neq 0, \\
Q(x, y)=B y^{K e}+\cdots+b_{1}(x) y+b_{0}(x), & B \neq 0 .
\end{array}
$$

With this representation the Newton-Puiseux roots at infinity $y(x)$ of each of the equations $P(x, y)=0$ and $Q(x, y)=0$ are fractional power series of the form

$$
y(x)=\sum_{k=0}^{\infty} c_{k} x^{1-k / m}, \quad m \in \mathbb{N}, \operatorname{gcd}\left\{k: c_{k} \neq 0\right\}=1,
$$

for which the map $\tau \mapsto\left(\tau^{m}, y\left(\tau^{m}\right)\right)$ is meromorphic and injective for $\tau$ large enough. In view of the Newton theorem we can represent

$$
P(x, y)=A \prod_{i=1}^{\operatorname{deg} P}\left(y-u_{i}(x)\right), \quad Q(x, y)=B \prod_{j=1}^{\operatorname{deg} Q}\left(y-v_{j}(x)\right),
$$

where $u_{i}(x)$ and $v_{j}(x)$ are the Newton-Puiseux roots at infinity of the equations $P=0$ and $Q=0$, respectively. We refer the readers to $[\mathrm{A}]$ and $[\mathrm{BK}]$ for the Newton theorem and the Newton-Puiseux roots.

We begin with the description of the non-proper value set $A_{f}$ of $f$ via Newton-Puiseux expansions. We will work with finite fractional power series $\varphi(x, \xi)$ of the form

$$
\varphi(x, \xi)=\sum_{k=1}^{K-1} a_{k} x^{1-k / m}+\xi x^{1-K / m}, \quad m \in \mathbb{N}, \operatorname{gcd}\left\{k: a_{k} \neq 0\right\}=1,
$$

where $\xi$ is a parameter. For convenience, we set $\operatorname{mult}(\varphi):=m$. Such a series $\varphi$ is called a dicritical series of $f$ if

$$
f(x, \varphi(x, \xi))=f_{\varphi}(\xi)+\text { lower order terms in } x, \quad \operatorname{deg} f_{\varphi}>0 .
$$

The following description of $A_{f}$ was given in [C]. 
Lemma 1 ([C, Theorem 4.4]).

$$
A_{f}=\bigcup_{\varphi \text { is a dicritical series of } f} f_{\varphi}(\mathbb{C}) .
$$

To see this, note that by definition the non-proper value set $A_{f}$ consists of all values $a \in \mathbb{C}^{2}$ such that there exists a sequence $\mathbb{C}^{2} \ni p_{i} \rightarrow \infty$ with $f\left(p_{i}\right) \rightarrow a$. If $\varphi$ is a dicritical series of $f$ of the form (7), we can define the map $\Phi(t, \xi):=\left(t^{-m}, \varphi\left(t^{-m}, \xi\right)\right)$. Then $\Phi$ sends $\mathbb{C}^{*} \times \mathbb{C}$ to $\mathbb{C}^{2}$ and the line $\{0\} \times \mathbb{C}$ to the line at infinity of $\mathbb{C P}^{2}$. The polynomial map $F_{\varphi}(t, \xi):=f \circ \Phi(t, \xi)$ sends the line $\{0\} \times \mathbb{C}$ to $A_{f} \subset \mathbb{C}^{2}$. Therefore, $f_{\varphi}(\mathbb{C})$ is an irreducible component of $A_{f}$, since $\operatorname{deg} f_{\varphi}>0$. Conversely, if $\ell$ is an irreducible component of $A_{f}$, one can choose a smooth point $\left(u_{0}, v_{0}\right)$ of $A_{f},\left(u_{0}, v_{0}\right) \in \ell$, and an irreducible branch at infinity $\gamma$ of the curve $P=u_{0}$ (or $Q=v_{0}$ ) such that the image $f(\gamma)$ is a branch curve intersecting $\ell$ transversally at $\left(u_{0}, v_{0}\right)$. Let $u(x)$ be the Newton-Puiseux expansion of $\gamma$ at infinity. Then we can construct a unique dicritical series $\varphi(x, \xi)$ such that $u(x)=\varphi\left(x, \xi_{0}+\right.$ lower order terms in $\left.x\right)$. For this dicritical series $\varphi$ we have $f_{\varphi}(\mathbb{C})=\ell$.

3. Associated sequence of a dicritical series. Let $\varphi$ be a given dicritical series of $f$. Let us represent it as

$$
\varphi(x, \xi)=\sum_{k=0}^{K-1} c_{k} x^{1-n_{k} / m_{k}}+\xi x^{1-n_{K} / m_{K}},
$$

where $0 \leq n_{0} / m_{0}<n_{1} / m_{1}<\cdots<n_{K-1} / m_{K-1}<n_{K} / m_{K}=n_{\varphi} / m_{\varphi}$ and $c_{i} \in \mathbb{C}$ may be zero, so that the sequence $\left\{\varphi_{i}\right\}_{i=0,1, \ldots, K}$ of series defined by

$$
\varphi_{i}(x, \xi):=\sum_{k=0}^{i-1} c_{k} x^{1-n_{k} / m_{k}}+\xi x^{1-n_{i} / m_{i}}, \quad i=0,1, \ldots, K-1,
$$

and $\varphi_{K}:=\varphi$ has the following properties:

(S2) For every $i<K$ at least one of the polynomials $p_{\varphi_{i}}$ and $q_{\varphi_{i}}$ has a root different from zero.

For every $\psi(x, \xi)=\varphi_{i}\left(x, c_{i}\right)+\xi x^{1-\alpha}, n_{i} / m_{i}<\alpha<n_{i+1} / m_{i+1}$, each of the polynomials $p_{\psi}$ and $q_{\psi}$ is either constant or a monomial in $\xi$.

The representation (8) of $\varphi$ is thus the longest representation such that for each $i$ there is a Newton-Puiseux root $y(x)$ of $P=0$ or $Q=0$ such that $y(x)=\varphi_{i}(x, c+$ lower order terms in $x)$ and $c \neq 0$ if $c_{i}=0$. This representation and the associated sequence $\left\{\varphi_{i}\right\}_{i=0,1, \ldots, K}$ are well defined and unique. Further, $\varphi_{0}(x, \xi)=\xi x$.

We will use the sequence $\left\{\varphi_{i}\right\}$ to determine the form of the polynomials $f_{\varphi}(\xi)$. For simplicity of notation, below we use lower indices " $i$ " instead of the lower indices " $\varphi_{i}$ ". 
For each $\varphi_{i}, i=0, \ldots, K$, let us write

$$
\begin{aligned}
& P\left(x, \varphi_{i}(x, \xi)\right)=p_{i}(\xi) x^{a_{i} / m_{i}}+\text { lower order terms in } x, \\
& Q\left(x, \varphi_{i}(x, \xi)\right)=q_{i}(\xi) x^{b_{i} / m_{i}}+\text { lower order terms in } x,
\end{aligned}
$$

where $p_{i}, q_{i} \in \mathbb{C}[\xi] \backslash\{0\}, a_{i}, b_{i} \in \mathbb{Z}$ and $m_{i}:=\operatorname{mult}\left(\varphi_{i}\right)$.

Let $\left\{u_{i}(x): i=1, \ldots, \operatorname{deg} P\right\}$ and $\left\{v_{j}(x): j=1, \ldots, \operatorname{deg} Q\right\}$ be the collections of the Newton-Puiseux roots of $P=0$ and $Q=0$, respectively. As shown in Section 2, by the Newton theorem the polynomials $P(x, y)$ and $Q(x, y)$ can be factorized as

$$
P(x, y)=A \prod_{i=1}^{\operatorname{deg} P}\left(y-u_{i}(x)\right), \quad Q(x, y)=B \prod_{j=1}^{\operatorname{deg} Q}\left(y-v_{j}(x)\right) .
$$

For each $i=0, \ldots, K$, define

$$
\begin{aligned}
& S_{i}:=\{k: 1 \leq k \leq \operatorname{deg} P, \\
&\left.u_{k}(x)=\varphi_{i}\left(x, a_{i k}+\text { lower order terms in } x\right), a_{i k} \in \mathbb{C}\right\}, \\
& T_{i}:=\left\{k: 1 \leq k \leq \operatorname{deg} Q, v_{k}(x)=\varphi_{i}\left(x, b_{i k}+\text { lower terms in } x\right), b_{i k} \in \mathbb{C}\right\}, \\
& S_{i}^{0}:=\left\{k \in S_{i}: a_{i k}=c_{i}\right\}, \quad T_{i}^{0}:=\left\{k \in T_{i}: b_{i k}=c_{i}\right\} .
\end{aligned}
$$

Write

$$
\begin{aligned}
& p_{i}(\xi)=A_{i} \bar{p}_{i}(\xi)\left(\xi-c_{i}\right)^{\# S_{i}^{0}}, \quad \bar{p}_{i}(\xi):=\prod_{k \in S_{i} \backslash S_{i}^{0}}\left(\xi-a_{i k}\right), \\
& q_{i}(\xi)=B_{i} \bar{q}_{i}(\xi)\left(\xi-c_{i}\right)^{\# T_{i}^{0},} \quad \bar{q}_{i}(\xi):=\prod_{k \in T_{i} \backslash T_{i}^{0}}\left(\xi-b_{i k}\right) .
\end{aligned}
$$

Lemma 2. (i) $n_{0}=0, m_{0}=1$ and

$$
\begin{array}{ll}
A_{0}=A, & \operatorname{deg} p_{0}=a_{0}=K d, \\
B_{0}=B, & \operatorname{deg} q_{0}=b_{0}=K e .
\end{array}
$$

(ii) For $i=1, \ldots, K$,

$$
\begin{aligned}
A_{i}= & A_{i-1} \bar{p}_{i-1}\left(c_{i-1}\right), \quad \operatorname{deg} p_{i}=\# S_{i}=\# S_{i-1}^{0}, \\
& \frac{a_{i}}{m_{i}}=\frac{a_{i-1}}{m_{i-1}}+\# S_{i-1}^{0}\left(\frac{n_{i-1}}{m_{i-1}}-\frac{n_{i}}{m_{i}}\right), \\
B_{i}= & B_{i-1} \bar{q}_{i-1}\left(c_{i-1}\right), \quad \operatorname{deg} q_{i}=\# T_{i}=\# T_{i-1}^{0}, \\
& \frac{b_{i}}{m_{i}}=\frac{b_{i-1}}{m_{i-1}}+\# T_{i-1}^{0}\left(\frac{n_{i-1}}{m_{i-1}}-\frac{n_{i}}{m_{i}}\right) .
\end{aligned}
$$

Proof. Note that $\varphi_{0}(x, \xi)=\xi x$ and $\varphi_{i}(x, \xi)=\varphi_{i-1}\left(x, c_{i-1}\right)+\xi x^{1-n_{i} / m_{i}}$ for $i>0$. Then, substituting $y=\varphi_{i}(x, \xi), i=0,1, \ldots, K$, into the Newton factorizations of $P(x, y)$ and $Q(x, y)$ in (11) one can easily verify the conclusions. 
4. The Jacobian condition. Let $\varphi$ be a dicritical series of $f$ and $\left\{\varphi_{i}\right\}$ be its associated series. Define

$$
J_{i}(\xi):=a_{i} p_{i}(\xi) \dot{q}_{i}(\xi)-b_{i} \dot{p}_{i}(\xi) q_{i}(\xi) .
$$

The Jacobian condition will be considered in the following sense.

LEMMA 3. Let $0 \leq i<K$. If $a_{i}>0$ and $b_{i}>0$, then

$$
J_{i}(\xi) \equiv \begin{cases}-m_{i} J(P, Q) & \text { if } a_{i}+b_{i}=2 m_{i}-n_{i}, \\ 0 & \text { if } a_{i}+b_{i}>2 m_{i}-n_{i} .\end{cases}
$$

Further, $J_{i}(\xi) \equiv 0$ if and only if $p_{i}(\xi)$ and $q_{i}(\xi)$ have a common root. In this case

$$
p_{i}(\xi)^{b_{i}}=C q_{i}(\xi)^{a_{i}}, \quad C \in \mathbb{C}^{*} .
$$

Proof. Since $a_{i}>0$ and $b_{i}>0$, differentiating $f\left(t^{-m_{i}}, \varphi_{i}\left(t^{-m_{i}}, \xi\right)\right)$ with respect to $t$, we obtain

$$
\begin{aligned}
m_{i} J(P, Q) t^{n_{i}-2 m_{i}-1}+ & \text { higher order terms in } t \\
& =-J_{i}(\xi) t^{-a_{i}-b_{i}-1}+\text { higher order terms in } t .
\end{aligned}
$$

Comparing the two sides we get the first conclusion. The remaining ones are left to the reader as an elementary exercise.

5. Proof of Theorem 1. (i) Assume that $A_{f} \neq \emptyset$. Then $A_{f}$ is a plane curve in $\mathbb{C}^{2}$. Let $\ell$ be an irreducible component of $A_{f}$. By Lemma 1 there is a dicritical series $\varphi$ of $f$ such that $\ell$ can be parameterized by the polynomial $\operatorname{map} f_{\varphi}(\xi)=\left(p_{\varphi}(\xi), q_{\varphi}(\xi)\right)$, i.e. $\ell=f_{\varphi}(\mathbb{C})$. We will show that

$$
f_{\varphi}(\xi)=\left(A C_{\varphi}^{d} \xi^{D_{\varphi} d}+\cdots, B C_{\varphi}^{e} \xi^{D_{\varphi} e}+\cdots\right), \quad C_{\varphi} \neq 0, D_{\varphi} \in \mathbb{N} .
$$

Then by changing variable $\xi \mapsto C_{\varphi}^{-1} \xi$ we get the desired parameterization $\xi \mapsto\left(A \xi^{D_{\varphi} d}+\cdots, B \xi^{D_{\varphi} e}+\cdots\right)$ of $\ell$. have

(ii) Consider the associated sequence $\left\{\varphi_{i}\right\}_{i=1}^{K}$ of $\varphi$. Since $A_{f} \neq \emptyset$, we

$$
\operatorname{deg} P>1, \quad \operatorname{deg} Q>1 .
$$

Otherwise, $f$ is bijective and $A_{f}=\emptyset$. Since $\varphi$ is a dicritical series of $f$, without loss of generality we can assume that

$$
\operatorname{deg} p_{K}>0, \quad a_{K}=0, \quad b_{K} \leq 0 .
$$

Then from the construction of the sequence $\varphi_{i}$ it follows that

$$
\begin{cases}p_{i}\left(c_{i}\right)=0 \text { and } a_{i}>0, & i=0,1, \ldots, K-1, \\ q_{i}\left(c_{i}\right)=0 & \text { if } b_{i}>0 .\end{cases}
$$

This allows us to use the Jacobian condition in the sense of Lemma 3. Then, by induction using Lemma 2, Lemma 3 and (13) we can obtain without difficulty the following. 
Assertion. For $i=0,1, \ldots, K-1$ we have

$$
\begin{gathered}
a_{i}>0, \quad b_{i}>0, \\
\frac{a_{i}}{b_{i}}=\frac{\# S_{i}}{\# T_{i}}=\frac{d}{e}, \\
\frac{\# S_{i}^{0}}{\# T_{i}^{0}}=\frac{d}{e}, \quad \bar{p}_{i}(\xi)^{e}=\bar{q}_{i}(\xi)^{d} .
\end{gathered}
$$

(iii) Now, we prove (12). By Lemma 2(iii) and (b-c) we have

$$
\begin{aligned}
\frac{b_{K}}{m_{K}} & =\frac{b_{K-1}}{m_{K-1}}+\# T_{K-1}^{0}\left(\frac{n_{K-1}}{m_{K-1}}-\frac{n_{K}}{m_{K}}\right) \\
& =\frac{e}{d}\left[\frac{a_{K-1}}{m_{K-1}}+\# S_{K-1}^{0}\left(\frac{n_{K-1}}{m_{K-1}} \frac{n_{K}}{m_{K}}\right)\right] \\
& =\frac{e}{d} \frac{a_{K}}{m_{K}}=0,
\end{aligned}
$$

as $a_{K}=0$. Hence, $f_{\varphi}(\xi)=\left(p_{K}(\xi), q_{K}(\xi)\right)$ by definition and (a). Using Lemma 2 (ii)-(iii) to compute the coefficients $A_{K}$ and $B_{K}$ we get

$$
A_{K}=A\left(\prod_{k \leq K-1} \bar{p}_{k}\left(c_{k}\right)\right), \quad B_{K}=B\left(\prod_{k \leq K-1} \bar{q}_{k}\left(c_{k}\right)\right) .
$$

Let $C_{\varphi}$ be a $d$ th root of $\prod_{k \leq K-1} \bar{p}_{k}\left(c_{k}\right)$ and $D_{\varphi}:=\operatorname{gcd}\left(\# S_{K-1}^{0}, \# T_{K-1}^{0}\right)$. Then, by Lemma 2 (ii) and (b-c) we have $A_{K}=A C_{\varphi}^{d}, B_{K}=B C_{\varphi}^{e}, \operatorname{deg} p_{K}=$ $\# S_{K-1}^{0}=D_{\varphi} d$ and $\operatorname{deg} q_{K}=\# T_{K-1}^{0}=D_{\varphi} e$. Thus,

$$
f_{\varphi}(\xi)=\left(A C_{\varphi}^{d} \xi^{D_{\varphi} d}+\cdots, B C_{\varphi}^{e} \xi^{D_{\varphi} e}+\cdots\right) .
$$

Acknowledgments. The author wishes to thank Prof. C. Olech and Prof. A. van den Essen for many valuable suggestions and useful discussions.

\section{References}

[A] S. S. Abhyankar, Expansion Techniques in Algebraic Geometry, Tata Inst. Fund. Research, 1977.

[AM] S. S. Abhyankar and T. T. Moh, Embeddings of the line in the plane, J. Reine Angew. Math. 276 (1975), 148-166.

[BCW] H. Bass, E. Connell and D. Wright, The Jacobian conjecture: reduction of degree and formal expansion of the inverse, Bull. Amer. Math. Soc. (N.S.) 7 (1982), 287-330.

[BK] E. Brieskorn und H. Knörrer, Ebene algebraische Kurven, Birkhäuser, Basel, 1981.

[C] Nguyen Van Chau, Non-zero constant Jacobian polynomial maps of $\mathbb{C}^{2}$, Ann. Polon. Math. 71 (1999), 287-310.

[E] A. van den Essen, Polynomial Automorphisms and the Jacobian Conjecture, Progr. Math. 190, Birkhäuser, Basel, 2000. 
[J] Z. Jelonek, The set of points at which a polynomial map is not proper, Ann. Polon. Math. 58 (1993), 259-266.

Hanoi Institute of Mathematics

18 Hoang Quoc Viet, 10307 Hanoi, Vietnam

E-mail: nvchau@math.ac.vn 